\title{
RESOURCE DIMENSIONING ASPECT OF HETEROGENEOUS TRAFFIC WITH DIFFERENT SERVICE REQIJIREMENTS: INTEGRATION VERSUS SEGREGATION ${ }^{1}$
}

\author{
Ramón Fabregat-Gesa, Jose Luis Marzo-Lázaro, Pere Ridao-Rodriguez ${ }^{2}$
}

\begin{abstract}
In this paper, we consider the ATM networks in which the Virtual Path concept is implemented. The question of how to multiplex two or more diverse traffic classes while providing different Quality of Service requirements is a very complicated, open problem. Two distint options are available: integration and segregation. In an integration approach all the traffic from different connections are multiplexed onto one VP. This implies that the most restrictive QOS requirements must be applied to all services. Therefore, link utilization will be decreased because unnecessarily stringent QOS is provided to all connections. With the segregation approach the problem can be much simplified if different types of traffic are separated by assigning a VP with dedicated resources (buffers and links). Therefore, resources may not the efficiently utilized because no sharing of bandwidth can take place across the VP. The probability that the bandwidth required by the accepted connections exceeds the capacity of the link is evaluated with the Probability of Congestion (PC). Since the PC can be expressed as the CLP, we shall simply carry out bandwidth allocation using the PC. We first focus on the influence of some parameters (CLP, bit rate and burstiness) on the capacity required by a VP supporting a single traffic class using the New Convolution Approach. Numerical results are presented both to ccompare the required capacity and to observe which conditions under each approach are preferred.
\end{abstract}

\section{INTRODUCTION}

In Asynchronous 'Transfer Mode (ATM) networks, different traffic classes with different flow characteristics and Quality of Sirvice (QOS) requirements are statistically multiplexed to increase the utilisation of link bandwidth.

QOS is normally expressed in terms of Cell Loss Probability (CLP), cell delay and jitter, in which both cell delay and jitter can easily be imposed by having a finite buffer to limit the maximum queue length. Therefore, we consider CLP requirements as QOS.

If statistical multijlexing is considered, the bandwidth required by the accepted connections could exceed the capacity of the link. The probability that the bandwidth required by the accepted connections exceeds the capacity of the link is evaluated by means of the Probability of Congestion (PC). Thus, PC, Total CLP and Individual CLP cas be calculated by using the New Convolution Approach (NCA).

In this paper, we consider those ATM networks in which the Virtual Path (VP) concept is implemented. How to multiplex two or more diverse traffic classes while providing different QOS requirements is a very complicated, open problem. Two distinct options are available: integration and segregation.

The paper is organised as follows. Section 2 describe the segregation and integration approach to virtual traffic management. In section 3 we present an algorithm to determine the minimum an sufficient capacity required by both approaches so that all the individual QOS requirements are guaranteed. In section 4 we explain how the

\footnotetext{
${ }^{1}$ This work has been supported by CICYT (Spanish Education Ministry) under contract TIC92-1289-PB.

${ }^{2}$ Departament d'Enginyeria Industrial. Universitat de Girona. Girona (Spain). Tel.: +34 72 418484. Fax +34 72

418399. E-mail (raraon,marzo)@ei.udg.es

(C) 1995 The Institution of Electrical Engineers

Printed and published by the IEE. Savoy Place, London WC2R OBL, UK.
} 
New Convolution Approach (NCA) computes the Individual Cell Loss Probabilities and how we can compute the Required Capacity in this case. Section 5 and 6 deal with numerical results, followed by conclusions and further works in Section 7.

\section{INTEGRATION VS. SEGREGATION}

When two traffic flows with different QOS requirements (i.e.: different Cell Loss Probabilities) arrive to a node and, these share the same destination node, as far as local decisions are concerned, two possible and simple approaches to virtual traffic management can be taken [1], [2], [3], [4], [5]:

In an INTEGRATION APPROACH ${ }^{3}$ (Sharing) all the traffic from different connections are multiplexed onto one VP. While sharing the available bandwidth and buffers. The capacity allocated to the VP should be large enough to satisfy all the individual QOS. Arriving cells from all source types are routed to a single FCFS buffer. In absence of buffer priorities, the same CLP is provided to both traffic types. This implies that the most restrictive QOS requirements must be applied to all services. Therefore, link utilisation will be decrease because unnecessarily stringent QOS is provided to all connections and efficient use of available resources is difficult since most source types receive a QOS that is much better than the one they had demanded. This fact could produce lower multiplexing gain than the segregation approach. The multiplexing gain is strongly dependent on the ratio between total link bandwidth and source mean bandwidth, concentrating a greater traffic intensity could conceivably produce a higher gain than segregation, even with the most stringent QOS requirements.

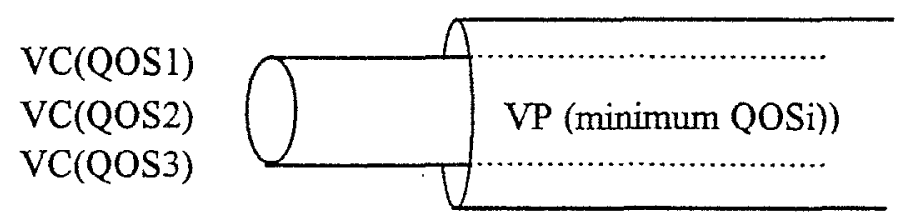

Figure 1a. Integration approach.

With the SEGREGATION APPROACH (Physical partitioning) the problem can be much simplified if different types of traffic are separated by assigning a VP with dedicated resources (buffers and bandwidth) tr each type of traffic. Arriving cells from different source types (or a set of source types) are directed to distinct FCFS buffers, which are then served in accordance to a chosen bandwidth assignment policy. Therefore, the resources may not be efficiently utilised because no sharing of bandwidth can take place across the VP. In this case, advantage can be taken on the less stringent QOS to achieve a high multiplexing gain. As a consequence of the separation, more than one VP may be established between the same source-destination pair, with each carrying different types of traffic. The capacity allocated to each VP should be sufficient to guarantee the required QOS of the traffic classes supported. The sum of all the assigned VP capacities would thus constitute the total capacity required.

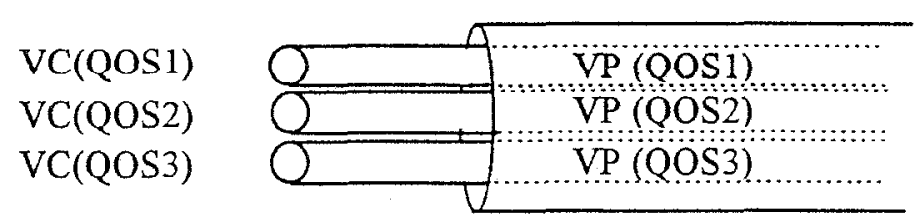

Figure 1b. Segregation approach.

\footnotetext{
${ }^{3}$ aggregation by some authors.
} 


\section{ALGORITHM TO DETERMINE THE MINIMUM CAPACITY REQUIRED BY A VIRTUAL PATH}

Superior performance of one or the other strategy depends on the particular characteristics of the involved traffic flows. There is a need of algorithms that, on the basis of small set of easily estimated parameters for each traffic flow, can predict accurately the benefits of aggregation / segregation.

In [6] we propose the New Convolution Approach to calculate the bandwidth required by different traffic types to achieve a certain QOS. When using this results, an iterative algorithm determines the sufficient and minimum capacity of a VP so that all the individual QOS are satisfied whenever different traffic classes are multiplexed together on the VP. The NCA must know the capacity, the number of sources of each type and the Individual CLP requirements by each type of traffic.

In [3], an efficient algorithm is developed to compute the minimum capacity required to satisfy all the QOS requirements when this classes of on-off sources are multiplexed onto a single VP. It applies the Markov Modulated Deterninistic Process method.

In order to decide: on integration/segregation, the different minimum link capacities needed for the multiplexing of the sources of each type must be computed.

We propose the below algorithm to determine the Minimum Capacity required so that all the individual QOS are guaranteed:

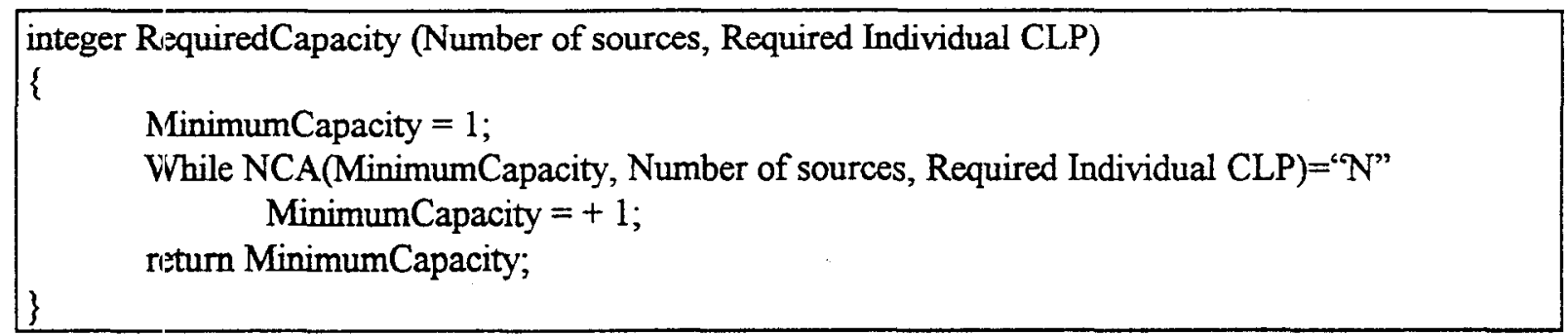

Algorithm to determine the minimum capacity required by a Virtual Path

By the segregation approach the minimum link capacity required by a link is the addition of the capacity required by each iype of traffic.

Capacity $=0$;

For $\mathrm{i}=1$ to Number of SourceTypes

Capacity $+=$ RequiredCapacity (Number of sources i, Required Individual CLP i);

\section{Segregation approach}

By the integration approach the NCA must be calculated by all the sources of all types and by the most restrictive QOS requirement: 
Capacity = RequiredCapacity (Number of sources i $\forall$ i, Minimum(Required Individual CLP i));

Most Restrictive Integration approach

The Required Capacity calculated with each strategy is taken as a basis for the decision.

\section{DIFFERENT SERVICE REQUIREMENTS BY INTEGRATION APPROACH IN ABSENCE OF PRIORITY BUFFERS}

If the network provides priority buffering mechanisms, different Cell Loss Probabilities can be satisfied by each traffic flow. Futhermore, in the integration approach it is not necessary that the most restrictive QOS requirements must be applied to all traffic classes

In absence of priority buffer, it is also possible to provide different Individual Cell Loss Probabilities by using the New Convolution Approach. In this case, it is neither necessary to apply the most restrictive QOS requirements.

\subsection{CAPACITY REQUIRED}

In this case the minimum link capacity required can be calculated with the NCA which allows that different Individual Cell Loss Probabilities to be satisfied by each traffic flow:

Capacity $=$ RequiredCapacity (Number of sources $i \forall$ i, Required Individual CLP $i \forall$ i));

Individual CLP Integration approach

\subsection{INDIVIDUAL CLP}

In this section we explain how the New Convolution Approach (NCA) computes the Individual Cell Loss Probabilities.

The NCA evaluates the statistical distribution of the instantaneous offered rate $Y$ by all established connections on a link. This permits the evaluation of the Probability of Congestion (PC). The PC is a parameter of the link state. It shows the probability that the resources of the link may be surpassed. We focused our study on the bandwidth of the link, that PC shows the probability that the offered rate $Y$ is larger than the capacity $C$ of the link. 


$$
P C(Y)=P(Y>C)=\sum_{L>C} P(Y=L)
$$

However, the PC does not give any information about the number of cells lost in case of congestion, Cell Loss Probability (CLP). In a short congestion state all cells may be buffered with no cell losses occurring. Nevertheless, when burst duration is longer than the size of the buffer, then almost all cells exceeding the link capacity are lost. In this case the relation between PC and CLP is approximated by:

$$
C L P(Y)=\frac{\sum_{L>C}(L-C) P(Y=L)}{E(Y)}
$$

Based on the NCA algorithm grouping connection in types, we propose the follow expression for the type-j cell loss probability:

$$
C L P_{j}=\frac{\sum_{L>C} \frac{L_{j}}{L}(L-C) P(Y=L)}{E\left(Y_{j}\right)}
$$

Where $L_{\mathrm{j}}$ is the rate offered by all type-j traffic when the instantaneous offered rate on the link is $L$ and $\mathrm{E}\left(Y_{j}\right)$ is the mean rate of all traffic of type-j. Both terms are easily obtained during the evaluation of PC based on NCA algorithm. To dernonstrate formula (3) it is necessary to sum individual CLP for all connections, so CLP can be evaluated by:

$$
C L P=\frac{\sum_{\forall j} E\left(Y_{j}\right) C L P_{j}}{E(Y)}
$$

Inserting the expression for $\mathrm{CLP}_{\mathrm{j}}$. we get

$$
\begin{gathered}
C L P=\frac{1}{E(Y)} \sum_{\forall j} E\left(Y_{j}\right) \frac{\sum_{L>C} \frac{L_{j}}{L}(L-C) P(Y=L)}{E\left(Y_{j}\right)} \\
C L P=\frac{1}{E(Y)} \sum_{\forall j} \sum_{L>C} \frac{L_{j}}{L}(L-C) P(Y=L)
\end{gathered}
$$

Interchanging the sums

$$
C L P=\frac{1}{E(Y)} \sum_{L>C} \sum_{\forall j} \frac{L_{j}}{L}(L-C) P(Y=L)
$$

But,

$$
\sum_{\forall j} L_{j}=L
$$

Resulting: 


$$
C L P=\frac{1}{E(Y)} \sum_{L>C}(L-C) P(Y=L)
$$

This is the expression for CLP as shows (1), and this implies that expression (3) is correct. Whenever NCA is used for CAC, individual CLPj can be an upper bound for bandwidth allocation.

\section{HOMOGENEOUS TRAFFIC}

In this section we investigate the influence of the peak bit rate and the mean bit rate on the capacity required by a VP to support a single traffic type (figure 2) and the Required Capacity for both integration and segregation approaches (figures 3-5).

\subsection{TRAFFIC MODEL}

We consider several types of on-off sources modelled by General Modulated Deterministic Process (GMDP). Each traffic types is characterised by the peak rate and by the probability of each state.

\begin{tabular}{|l|c|c|c|c|c|c|}
\hline traffic type & \multicolumn{2}{|c|}{ A } & \multicolumn{2}{c|}{ B } & \multicolumn{2}{c|}{ C } \\
\hline & rate & prob. & rate & prob. & rate & prob. \\
\hline state 0 & 10 & 0.1 & 5 & 0.1 & 5 & 0.02 \\
\hline state 1 & 0 & 0.9 & 0 & 0.9 & 0 & 0.98 \\
\hline mean rate & \multicolumn{2}{|c|}{1} & \multicolumn{2}{|c|}{0,5} & \multicolumn{2}{c|}{0,1} \\
\hline burstiness & \multicolumn{2}{|c|}{10} & \multicolumn{2}{|c|}{10} & \multicolumn{2}{c|}{50} \\
\hline
\end{tabular}

Traffic types.

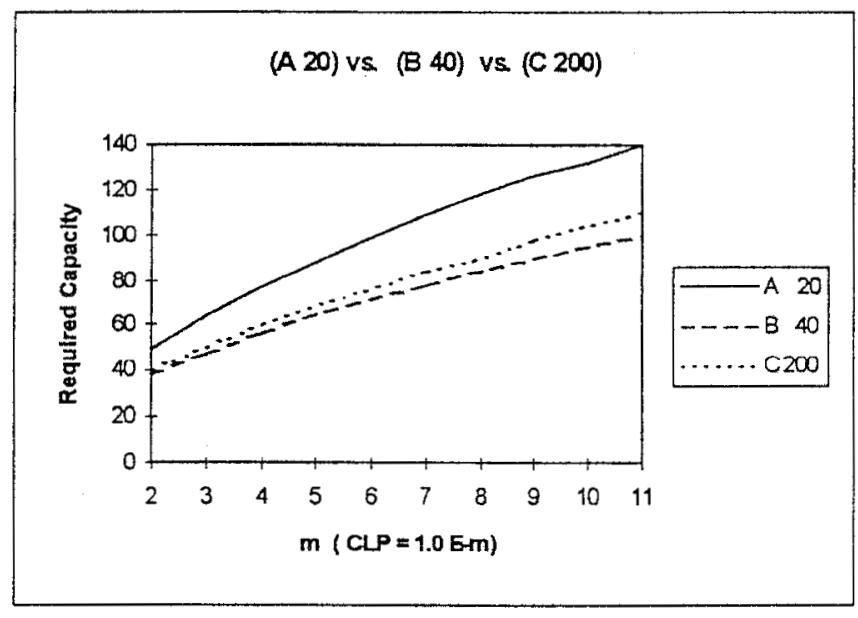

Figure 2 : Required Capacity vs. Individual CLP
Fig. 2 shows the Required Capacity varying the QOS requirements (Individual CLP) from $1 \mathrm{E}-2$ to $1 \mathrm{E}-11$, for three types of sources. We consider $2 C$ connections of type A, 40 connections of type B and 200 connections of type C. This number of connections has been chosen in order that the mean load of the system were the same in the three cases.

For the same burtiness a low number of type A connections demand more bandwidth than type B connections. If there are few connections on a link the statistical multiplexing gain may be small.

Type $\mathrm{C}$ connections demand more bandwidth than type $\mathbf{B}$ connections due to the fact that the burstiness of Type $C$ connections is larger than the burstiness of Type B connections.

All type of sources demand more bandwidth whenever the QOS is more stringent.

In figures 3, 4 and 5 we compare the segregation approach whit the integration approach when one type of connections is considered only. Results are plotted variyng the Individual CLP ( $\mathrm{n}$ and $\mathrm{m}$ ) from $1 \mathrm{E}-2$ to $1 \mathrm{E}-11$. 
Figures 3a, 4a ancl 5a show the Required Capacity when using segregation approach and integration approach. In each figure only one type of connections are considered ("homogeneous traffic"). By the segregation one, two VP are considerecl; each with the same number of connections (NC). By the integration one, there is one VP with double number of connections $(2 * \mathrm{NC})$. Dotted lines show the Required Capacity using the segregation approach and solid lines show the Required Capacity using the integration approach. In figures ' $a$ ', the upper line corresponds tio $\mathbf{n}=11$. The horizontal solid lines result from the fact that in the integration approach the Required Capacity' is solely determined by the most stringent QOS requirement.

The load of system is the same in all the cases, and it is the double of the considered in figure 2.

Figures $3 b, 4 b$ and $5 b$ show the reduction of the Required Capacity when the integration approach is used instead of the segregation approach. In this case, figures $b$, when $m=2$ the upper line corresponds to $n=11$, but when $\mathrm{m}=11$ it corresponds to $\mathrm{n}=2$. When the results are greater than 0 the integration approach is better; otherwise the segregation one is better.

These results show that :

- the integration approach requires less capacity than the segregation approach almost in all cases,

- when one Individual CLP (n) is fixed, the greatest reduction of the Required Capacity is obtained when the other Individual CLP (m) are the same value, i.e. $\mathrm{n}=\mathrm{m}$.

- if $n=-m$, the reduction of the Required Capacity increases when the Individual CLP is more stringent (n increases)

Figures $3 \mathrm{a}$ and $3 \mathrm{~b},(\mathrm{~B} 40)+(\mathrm{B} 40$ ) vs. (B 80) show that in the segregation approach we consider 40 connections of type B by each VP, where as in the integration approach we consider 80 connections of type B. The Individual CL.P is $\mathrm{m}$ for first connections, and $\mathrm{n}$ for second connections.

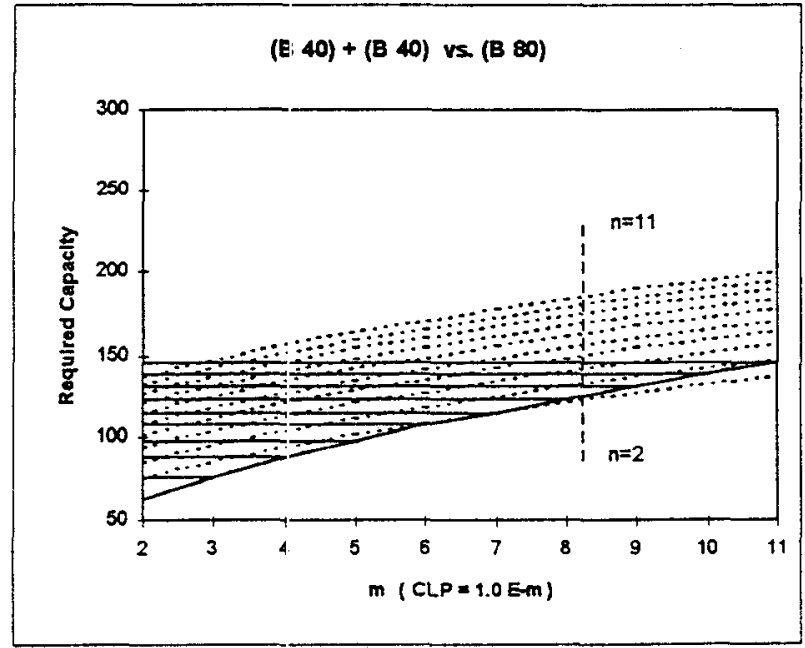

Figure 3a: Recuired Capacity vs. Individual CLP

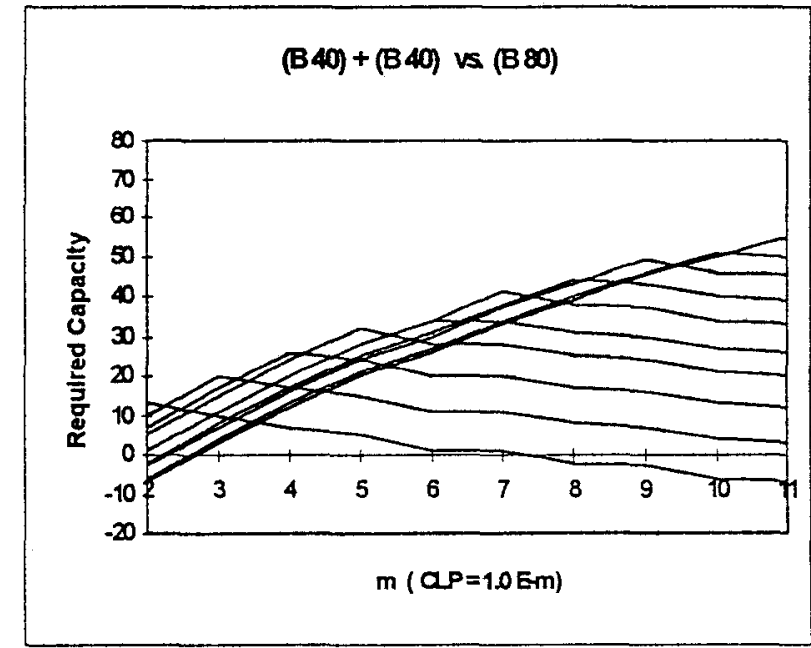

Figure 3b: Reduction of the Required Capacity vs. Individual CLP

By $\mathrm{NC}=40$ connections of type $\mathrm{B}$, the integration approach requires less capacity than the segregation approach for almost all cases, except for the cases of: $n=2$ and $m>7$, or $n>7$ and $n=2$. 


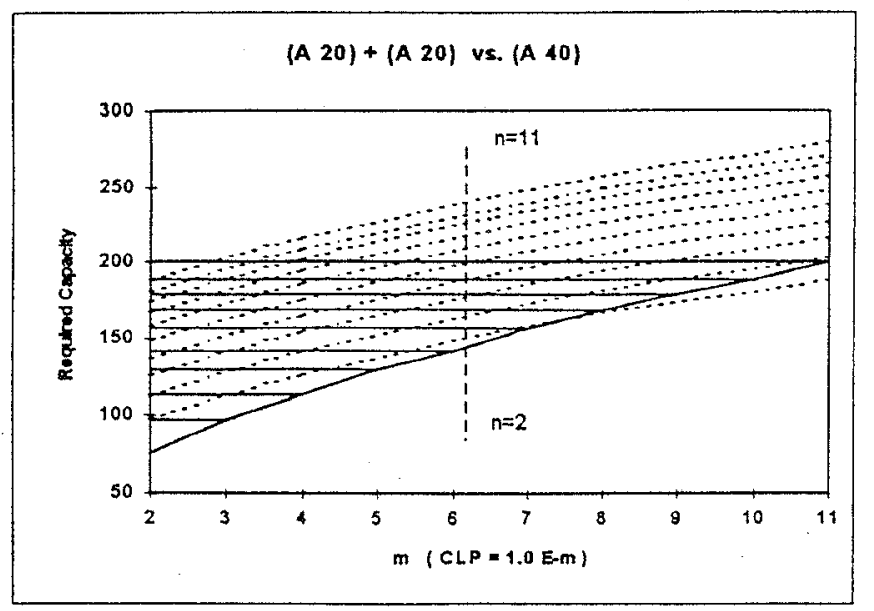

Figure 4a: Required Capacity vs. Individual CLP

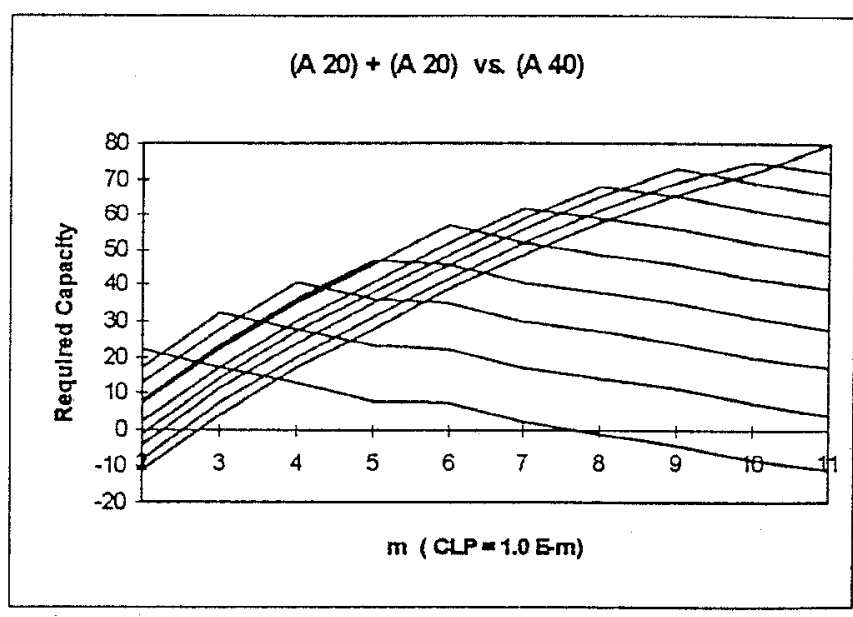

Figure 4b: Reduction of the Required Capacity vs. Individual CLP

By $\mathrm{NC}=20$ connections of type $\mathrm{A}$, the integration approach requires less capacity than the segregation approach for almost all cases, except for the cases of: $n=2$ and $m>7$, or $n>7$ and $n=2$.

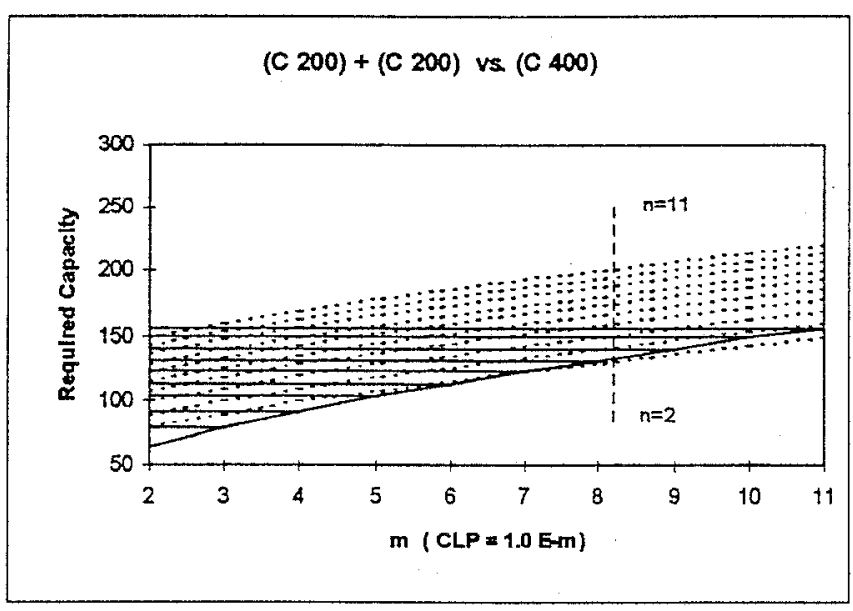

Figure 5a: Required Capacity vs. Individual CLP

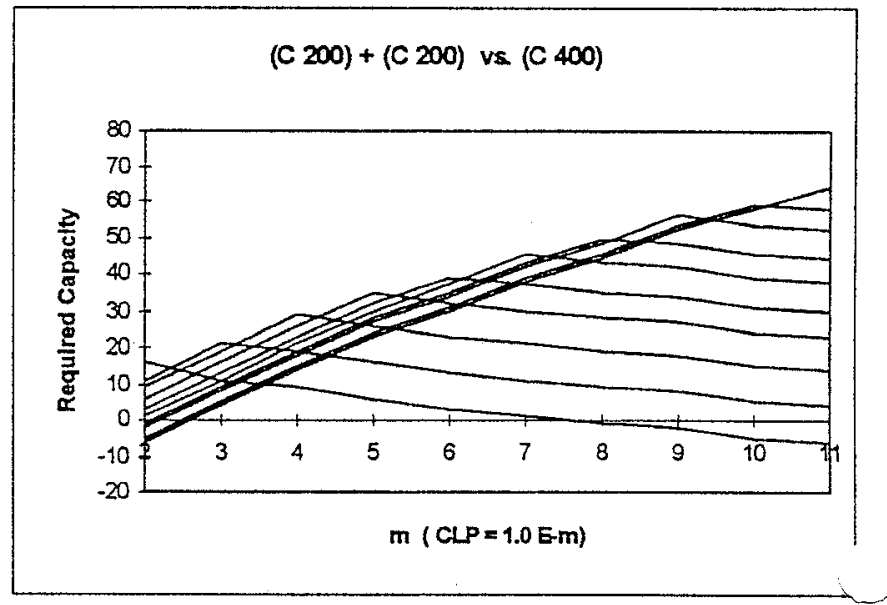

Figure 5b: Reduction of the Required Capacity vs. Individual CLP

By $\mathrm{NC}=20$ connections of type $\mathrm{A}$, the integration approach requires less capacity than the segregation approach for almost all cases, except for the cases of: $n=2$ and $m>7$ or $n>7$ and $n=2$.

\section{HETEROGENEOUS TRAFFIC}

In this section we compare the Required Capacity for integration and segregation approaches when two type of connections are considered. The load of system is the same in all the cases, and it is the same that we have considered in the figures below.

Figures $6 a$ and 6b, (B 40) + (A 200) vs. (B 40, A 200) show that in the segregation approach we consider 40 connections of type B by one VP and 200 connections of type A by the other, where as in the integration approach we consider in the same VP 40 connections of type B and 200 connections of type A. In this case, the Individual CLP is $m$ for type $B$ connections and $n$ for type $A$ connections. 


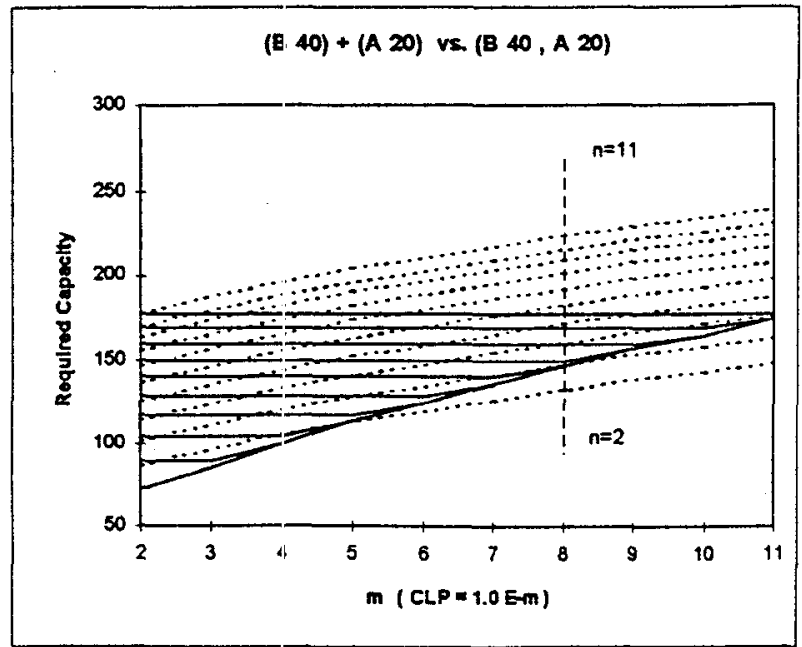

Figure 6a: Rexuired Capacity vs. Individual CLP

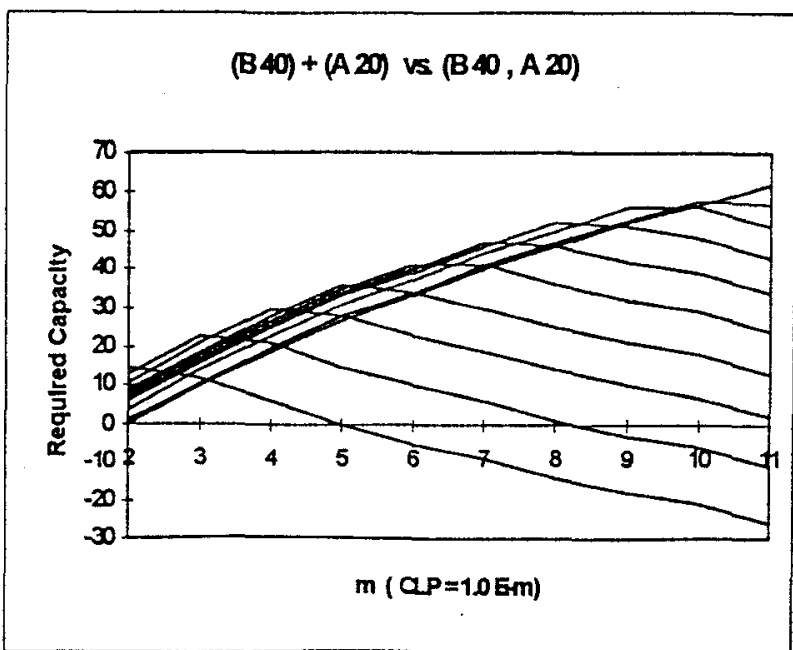

Figure 6b: Reduction of the Required Capacity vs. Individual CLP

By $N C=40$ connections of type $B$ and $N C=20$ connections of type $A$, the integration approach requires less capacity than the segregation approach for almost all cases, except for the cases of: $n=2$ and $m>5$ or $n=3$ and $\mathrm{m}>8$.

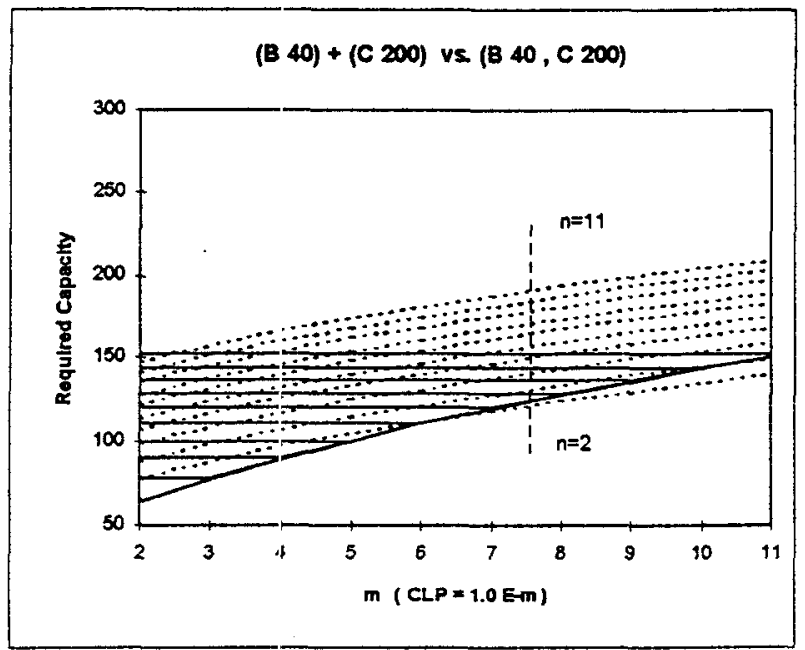

Figure 7a: Rerpuired Capacity vs. Individual CLP

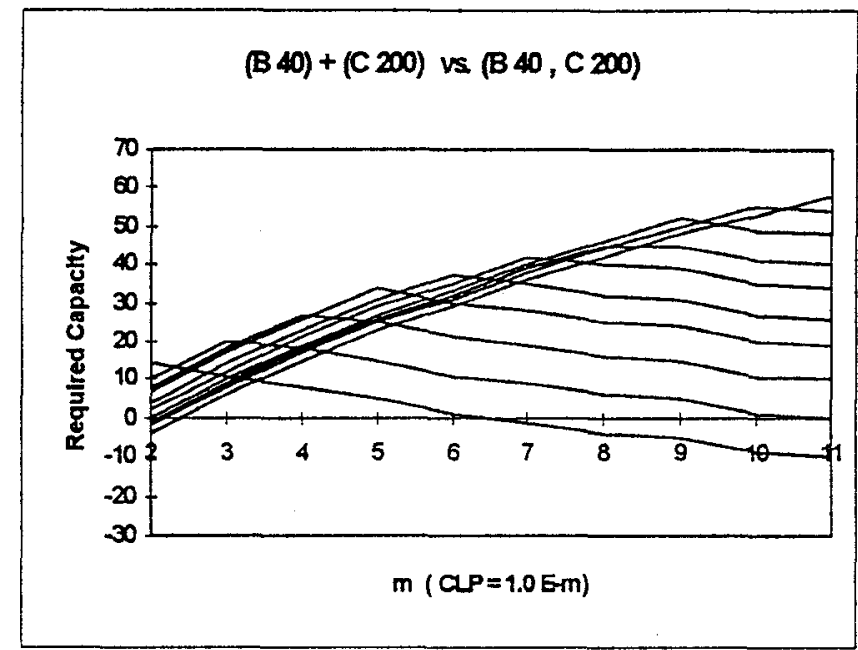

Figure 7b: Reduction of the Required Capacity vs. Individual CLP

By NC $=40$ connections of type $B$ and $N C=200$ connections of type $C$, the integration approach requires less capacity than the segregation approach for almost all cases, except for the cases of: $n=2$ and $m>6$

By $\mathrm{NC}=40$ connections of type $\mathrm{B}$ and $\mathrm{NC}=20$ connections of type $\mathrm{A}$, the integration approach requires less capacity than the segregation approach in all cases.

Solid lines are not completely horizontal because the Required Capacity is plotted for Individual CLP integration approach. The Individual CLP integration approach requires less or same capacity that the Most Restrictive integration approach in all cases. Therefore, this improvement implies that segregation approach is not the best in sorne cases. 


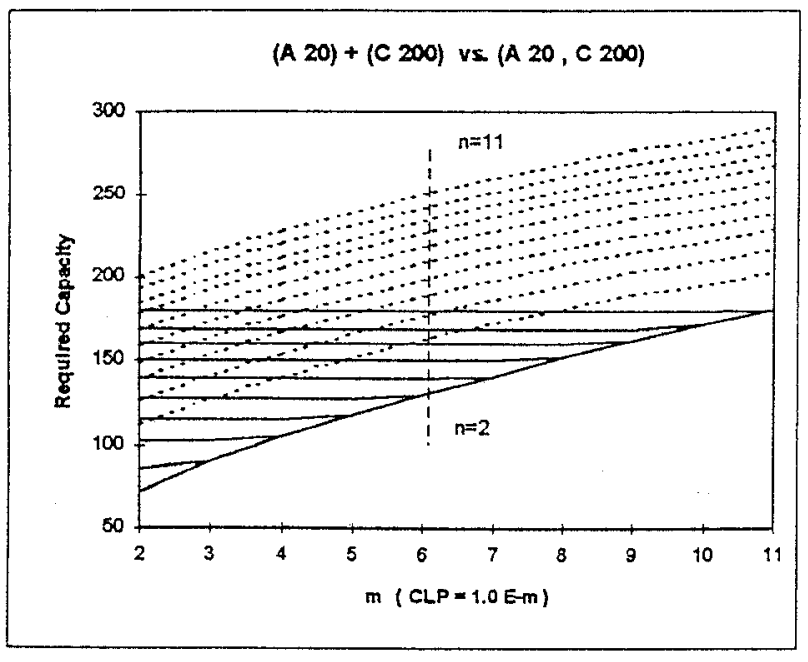

Figure 8a: Required Capacity vs. Individual CLP

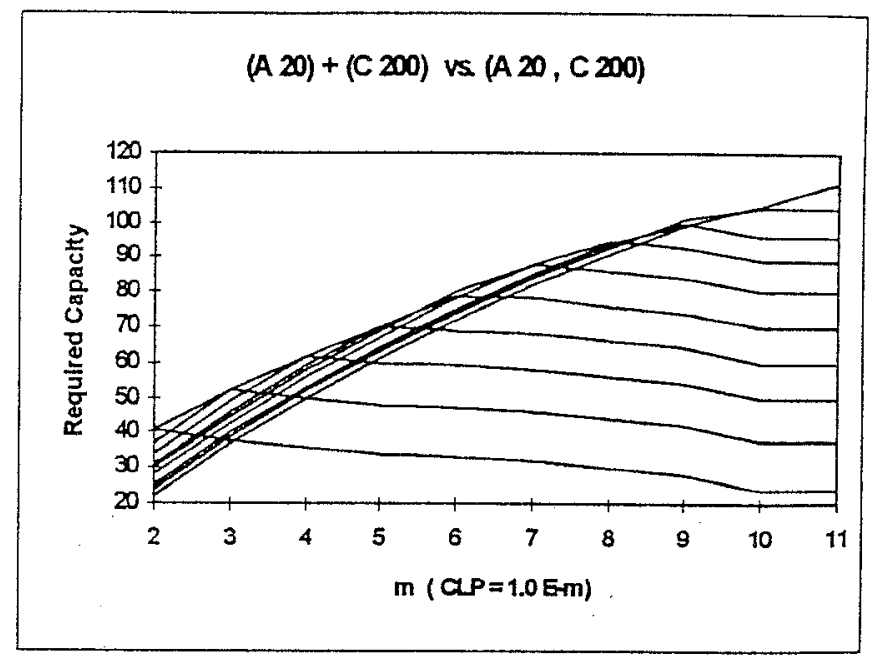

Figure 8b: Reduction of the Required Capacity vs. Individual CLP

\section{CONCLUSIONS}

In this paper, we present a comparison between integration and segregation when the concept of Virtual Path is implemented in ATM networks. We introduce a new method to evaluate the required bandwidth. This method is based on the New Convolution Approach and evaluates the Probability of Congestion in a link or VP.

When using integration this method resources may be more efficiently utilized. Numerical results are presented both to compare the required capacity and to observe which conditions under each approach are preferred. This results show that the integration approach requires less capacity than the segregation approach for almost all cases and the Individual CLP integration approach requires less or same capacity that the Most Restrictive integration approach in all cases

We are continuing our work considering more complex scenarios varying the load and the amount of VP'r considered

\section{REFERENCES}

[1] Management Committee of the COST 224 Project. "Cost 224 Final Report: Performance Evaluation and Design of Multiservice Networks

[2] S. Gupta and M. El Zarki. "Traffic Classification for Round-Robin Scheduling Schemes in ATM Networks". INFOCOM'93, pp. 820-827

[3] J.H.S. Chan and D.H.K. Tsang. "Bandwidth Allocation of Multiple QOS Classes in ATM Environment" INFOCOM'94, pp. 360-367

[4] R.H. Hwang, J.E. Kurose and D. Towsley. "MDP Routing in ATM Networks Using the Virtual Path Concept".INFOCOM'94. pp. 1509-1517

[5] U. Mocci and C. Scoglio. "Traffic Clustering Rules in ATM Networks". GLOBECOM'94, pp. 783-787.

[6] R. Fabregat-Gesa, J. Sole-Pareta, J.L. Marzo-Lázaro and J. Domingo-Pascual. "Bandwidth Allocation Based on Real Time Calculations Using the Convolution Approach". GLOBECOM'94. 